\title{
Application of Paper Folding Technique to Three-Dimensional Space Sound Absorber with Permeable Membrane: Case Studies of Trial Productions
}

\author{
Kimihiro Sakagami and Takeshi Okuzono \\ Environmental Acoustics Laboratory, Department of Architecture, Graduate School of Engineering, Kobe Univer- \\ sity, Rokko, Nada, Kobe 657-8501, Japan.
}

\begin{abstract}
Hirotaka Suzuki and Nao Koyanagi
Lighting Environment Planning Laboratory, Department of Architecture, Graduate School of Engineering, Kobe University, Rokko, Nada, Kobe 657-8501, Japan.
\end{abstract}

\begin{abstract}
Masahiro Toyoda
Department of Architecture, Faculty of Environmental and Urban Engineering, Kansai University, Yamate-cho, Suita, Osaka 564-8680, Japan.
\end{abstract}

\begin{abstract}
(Received 26 August 2019; accepted 19 December 2019)
The authors propose a space sound absorber made of a permeable membrane (PM), including woven or nonwoven fabrics in three-dimensional shapes, e.g., cylinder, rectangle, etc. The proposed absorbers are examined by experimental measurements and boundary element analyses, and it is found that they can be effectively used especially for middle and high frequencies. In order to develop these absorbers for wider applications, it would be desirable to give them additional values and functions, particularly to elaborate on their design. Supposing that they could also be used for lighting equipment, such as lampshades as one of the applications, pilot studies on pseudo-cylindrical, and pseudo-spherical PM space absorbers with uneven surfaces produced by paper-folding (origami technique) are carried out. The pseudo-cylindrical concave curves (PCCC) shell shape has been proven as a suitable form for a lampshade, and the pseudo-spherical concave curves (PSCC) shell shape is an application of PCCC. In this paper, PCCC and PSCC shell shapes are applied to three-dimensional PM space absorbers, and trials are conducted using PMs selected by flow resistance measurements and preliminary simulations. The sound absorptivity of the specimens is measured in a reverberation chamber, and their absorptivity is 0.6 (PCCC case) and 0.4 (PSCC case) at mid-high frequencies.
\end{abstract}

\section{INTRODUCTION}

The sound absorption effect of permeable membranes (PMs), including woven and non-woven fabrics, has been extensively studied for many years. ${ }^{1}$ The acoustical effect of textiles and fabrics is widely appreciated. ${ }^{2}$ Fabrics and textiles are also widely appreciated for their high recyclability. ${ }^{2}$ The results of studies on various types of conventional sound absorbing systems with PMs are widely known. ${ }^{1}$ There have also been detailed investigations into the acoustic properties of PMs, in a series of studies on different types of sound absorbing systems with permeable membranes. ${ }^{3,4}$

Recently, the authors proposed space sound absorbing systems using PMs, including textiles and woven or non-woven fabrics, which are constructed in a three-dimensional object such as a cylinder, rectangle, or plane, and studied their sound absorbing characteristics experimentally as well as analytically and numerically with boundary element method (BEM) simulations. ${ }^{5-7}$ The results showed that these membrane space sound absorbers offer moderate sound absorptivity, about 0.5 0.6 at maximum, in the middle to high frequency ranges, depending on the absorber's parameters and conditions. Therefore, it was confirmed that these space absorbers can be effectively used in some situations.
One potential application of three-dimensional space sound absorbers with permeable membrane (3-D PMA) is lighting equipment such as lampshades. For this purpose, more elaborate design of the surface texture and shape is desirable to offer a variety of designs.

In this paper, the preliminary results of our pilot study of a sound absorbing lampshade made of permeable membrane with the paper folding technique for more designable shapes are introduced. The paper folding (or origami) technique has also recently been applied for noise barrier design; ${ }^{8}$ also, the sound absorption of a "Sensu" (folding fan) shaped paper has been studied in detail with both theoretical and experimental approaches. ${ }^{9}$ This paper studies the sound absorptivity of folded paper of a Japanese folding fan (Sensu) shape. Papers are, in many cases, not permeable, but the attenuation by the boundary layer friction between surfaces was considered instead. This study will provide a physical insight into the acoustic effect of folded materials; however, to the authors' knowledge, there is no research work on the application of paper folding technique to permeable membranes for sound absorbing structures. Therefore, the intention of this work is to provide a basic discussion on its feasibility through an experimental case study. 




Figure 1. Cylindrical lamp shade of pseudo-cylindrical concave polyhedral (PCCP) shell (left), pseudo-cylindrical concave curves (PCCC) shell (centre), and simple cylinder (right) (after Suzuki). ${ }^{9}$ In the case of the PCCC shell shape, luminance distribution gradually changes on the surface.

\section{CYLINDRICAL PM ABSORBER WITH PAPER FOLDING TECHNIQUE (PCCC SHELL 3-D PMA)}

\subsection{Pseudo-cylindrical Concave Curves (PCCC) Shell Shape}

Suzuki studied the application of the paper folding method, which was recently applied to lampshade design, to cylindrical shells. ${ }^{10}$ He studied cylindrical shells with non-planar quadrilateral units of non-flat texture on the surface for lampshade design. Similar shapes have been known as the Yoshimura pattern or pseudo-cylindrical concave polyhedral (PCCP) shell; ${ }^{11,12}$ Suzuki proposed a pseudo-cylindrical concave curves (PCCC) shell, which is a variant of the PCCP shell made by removing valley fold lines. Suzuki proved that the PCCC shell is better in both luminescence distribution and designability for lampshades (see Fig. 1). ${ }^{10}$

This shape is applicable to 3-D PMA, and is particularly easy to apply to cylindrical 3-D PMA, to offer better designability and luminescence distribution for the aesthetic benefit of sound absorbing technique. . $^{6}$

\subsection{PCCC Space Sound Absorber with PMs}

\subsubsection{Selection of the material}

Regarding the material for the trial production of the PCCC shell PM space sound absorber, it is necessary to select a permeable membrane that can offer enough sound absorption effect. It is known that the optimal flow resistance is twice the air impedance $(2 \rho \mathrm{c}){ }^{3}$ In the present work, the PCCC shell was produced by making a folding line in the membrane, and processability should be considered. Considering the latter point, a PM with a coating, which was used in the previous study, is not suitable as it is not foldable. ${ }^{4}$ Therefore, in this study, we prepared three kinds of uncoated PMs and measured their flow resistance and surface density. The results are listed in Table 1. Consequently, a woven textile made of fiberglass with a flow resistance of $1200 \mathrm{~Pa} \mathrm{~s} / \mathrm{m}$ and surface density of $0.205 \mathrm{~kg} / \mathrm{m}^{2}$ was selected.

\subsubsection{Preliminary check of selected material by BEM simulation}

The material for the trial production was thus selected. However, comparing it with the most efficient material used in our previous study $\left(0.495 \mathrm{~kg} / \mathrm{m}^{2}\right)$, the material selected above had a smaller surface density. ${ }^{4}$ Light weight membranes
Table 1. Measured flow resistance and surface density of PMs tested for the selection for trial production of PCCC 3-D PMA specimen.

\begin{tabular}{|c|c|c|}
\hline & $\begin{array}{c}\text { Flow resistance } \\
(\mathrm{Pa} \mathrm{s} / \mathrm{m})\end{array}$ & $\begin{array}{c}\text { Surface density } \\
\left(\mathrm{kg} / \mathrm{m}^{2}\right)\end{array}$ \\
\hline PET woven fabric 1 & 105 & 0.08 \\
\hline PET woven fabric 2 & 80 & 0.08 \\
\hline Fiberglass textile & 1200 & 0.205 \\
\hline
\end{tabular}

tended to show lower sound absorptivity even if the flow resistance was comparable. ${ }^{4}$ Therefore, in order to determine the expected absorptivity value of the test specimen, we made a BEM simulation for a cylindrical 3-D PMA (without folding) to give a first approximation of expected absorptivity of the test specimens made of the selected material. ${ }^{6}$ The method of the BEM simulation was detailed in Toyoda et al. ${ }^{6}$

As is shown in Fig. 5 of subsequent section, the result of the simulation, with the parameters of the selected material above, showed that the selected material can offer moderately high sound absorptivity, i.e., around 0.5 to 0.6 at middle and high frequencies. Thus, by the test specimen with the material, though it was rather light-weight, a reasonable sound absorption effect can be expected. Therefore, we decided to use the material for the trial production.

\subsubsection{Procedure of trial production of PCCC shell 3-D PMA}

The result of the preliminary consideration in the preceding section showed the possibility to obtain effective absorption performance by using the selected material. Therefore, we proceeded to the trial production of PCCC shell 3-D PMAs with the material. For comparison purposes, cylindrical 3-D PMAs without folding were produced with the same material. Schematic representations of these specimens are shown in Fig. 2 and photographs are shown in Fig. 3. The cylindrical 3-D PMA (without folding (a)) and PCCC shell 3-D PMA (b) were both made from the material cut into rectangles $85.3 \mathrm{~cm}$ (except for the edges) by $117.3 \mathrm{~cm}$ in size. The production processes for (a) and (b) are as follows:

(a) For cylindrical 3-D PMA, the cut material was rounded to a cylindrical shape. The triangular edges on the top and bottom were folded to fix the material to the wire frame on the top and bottom of the absorber. The diameter was $37.3 \mathrm{~cm}$, height was $85.2 \mathrm{~cm}$, and surface area was $1 \mathrm{~m}^{2}$.

(b) For the PCCC shell 3-D PMA, before the cut material was rounded, folding lines were made by an iron. After this procedure, it was rounded to shape a cylinder, and diamond-shaped hollows were made by hand. The diameter was $37.3 \mathrm{~cm}$, height was $83.2 \mathrm{~cm}$, and surface area was $1 \mathrm{~m}^{2}$.

As mentioned above, for both (a) and (b), except for the triangular edges to fix the material to the wire frame (to keep the circular sectional shape), the surface area was $1 \mathrm{~m}^{2}$. Both (a) and (b) can stand alone without support. The inside was empty, and the top of the specimen was open, as the absorption characteristics did not change significantly by closing it. ${ }^{5}$ In both cases, the surface area was the same, but PCCC shell type (b) was shorter because of the folding lines. The depth of the hollows on the surface of PCCC shell type (b) was $10 \mathrm{~mm}$ at the maximum. We prepared five specimens of each type for the experiment. 




Figure 2. Sketch of specimens. Membrane material is cut out as shown (left) and shaped into a cylindrical form (right) for simple cylindrical absorber (without folding, top) and PCCC shell absorber (bottom).

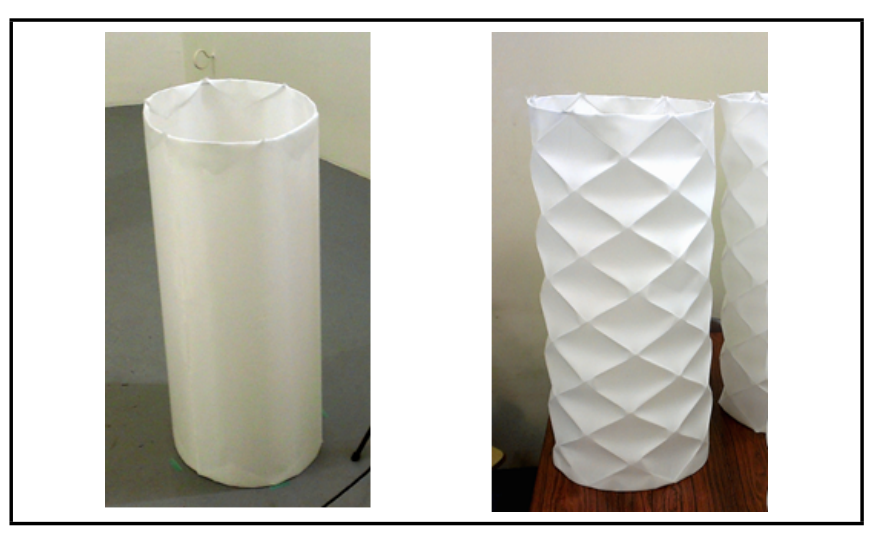

Figure 3. Photographs of absorber specimens: cylindrical three-dimensional space sound absorber with permeable membrane (3-D PMA) without folding (left), and PCCC shell 3-D PMA (right).

\subsection{Measurement and Results}

We conducted our experimental measurements in a reverberation chamber in accordance with JIS A 1409 (ISO 354 compatible). In the experiment, as explained above, the surface areas of both samples were the same $\left(1 \mathrm{~m}^{2}\right)$, and five specimens were placed on the rigid floor of the reverberation chamber (volume: $317.4 \mathrm{~m}^{3}$; surface area: $282.3 \mathrm{~m}^{2}$ ) as shown in Fig. 4. A noise interrupting method was used with one sound source and three microphone positions. The results are shown in Fig. 5, compared with the measured results of a cylindrical 3-D PMA and its theoretical result by preliminary BEM simulation (Sec. 2.2.2). It showed that a PCCC 3-D PMA gave almost the same results as a cylindrical 3-D PMA (without folding). This meant that folding was useful for designability and lighting performance, but did not affect the sound absorbing performance significantly. Therefore, PCCC shell 3D PMA can be effectively applicable to PM sound absorbing lampshades.

\section{SPHERICAL PM ABSORBER WITH PAPER FOLDING TECHNIQUE (PSCC SHELL 3-D PMA)}

\subsection{Expected Effect of Spherical Space Sound Absorber with PMs}

Considering the shape of 3-D PMA, it had been proven that a cylinder was more efficient in absorption performance than

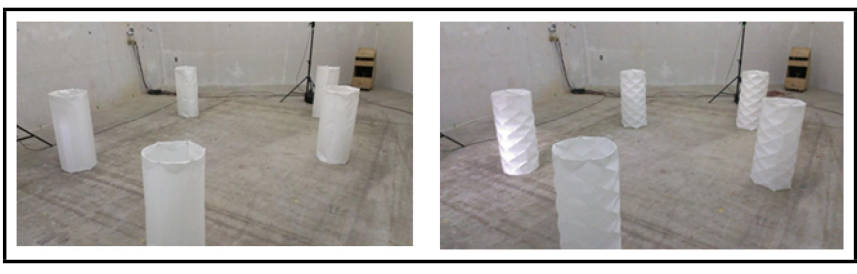

Figure 4. Specimens arranged in reverberation chamber: cylindrical 3-D PMAs (left); PCCC 3-D PMAs (right).

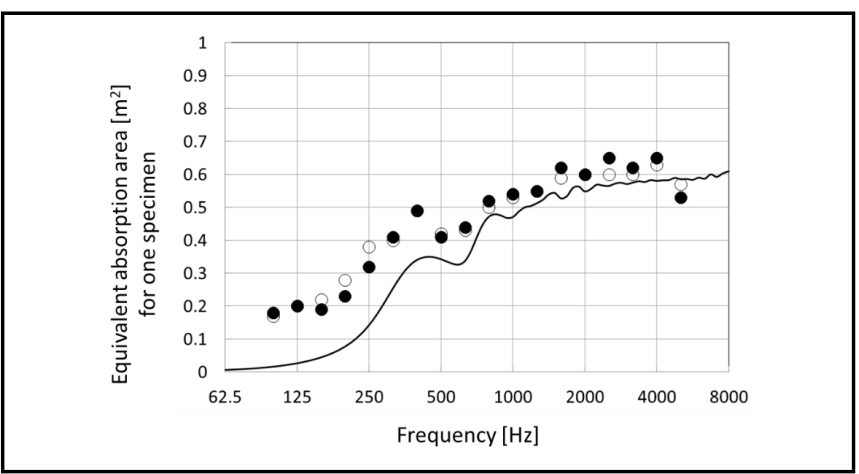

Figure 5. Example of equivalent absorption area for one specimen measured in a reverberation chamber of PCCC 3-D PMA (filled circle) compared with cylindrical 3-D PMA (open circle). Solid line shows theoretical values calculated by BEM simulation for cylindrical type (without folding) in the preliminary consideration (Sec. 2.2.2) for comparison.

a rectangle, because the incidence wave impinged normally at any point on the surface in the case of a cylinder. ${ }^{5}$ This was observed for both 3-D PMA and 3-D microperforated panel (MPP) space absorbers. ${ }^{5-7,13,14}$ Usually permeable materials show better sound absorption performance in normal incidence conditions. Inferring from these results, a discussion led to the idea that a spherical absorber should also be efficient.

In order to produce a spherical 3-D PMA, a similar shape to PCCC with folding technique was applicable to a spherical shape, i.e., pseudo-spherical concave curves (PSCC). In this report, a trial production of a 3-D PMA with PSCC shell shape was carried out, and its sound absorption performance was measured in a reverberation chamber. A similar shape with a triangular folding pattern was applied to commercially available lampshades; ${ }^{15}$ however, the PSCC shell shape had a rectangular folding pattern, which was different from the existing products. Although only one type of trial production was made, it was compared with cylindrical 3-D PMA without folding to discuss the effect of its shape and unevenness.

\subsection{Material Used in this Study}

In this trial production, the material used for the specimen should be suitable for both sound absorbing purposes and lampshades, i.e., a PM with acoustic permeability and light transmissibility. Besides, to apply the PSCC shell shape approach, for ease of folding, we examined three non-woven polypropylene (PP) listed in Table 2. We selected "PP nonwoven 2" with flow resistance of $64 \mathrm{~Pa} / \mathrm{s}$ and surface density of $0.125 \mathrm{~kg} / \mathrm{m}^{2}$ among three samples considering processability. The light transmittance of this material was desirable for a lampshade; however, the flow resistivity was rather lower than the optimal value for sound absorbing purposes while keeping the best processability. ${ }^{3}$ Therefore, in this trial production, we prioritized processability for folding to make a good shape as a PSCC shell shape lampshade. 
Table 2. Measured flow resistance and surface density of PMs tested for the selection for trial production of PSCC 3-D PMA specimen.

\begin{tabular}{||c|c|c|}
\hline & Flow resistance $(\mathrm{Pa} \mathrm{s} / \mathrm{m})$ & Surface density $\left(\mathrm{kg} / \mathrm{m}^{2}\right)$ \\
\hline PP non-woven 1 & 19 & 0.07 \\
\hline PP non-woven 2 & 64 & 0.125 \\
\hline PP non-woven 3 & 221 & 0.24 \\
\hline
\end{tabular}

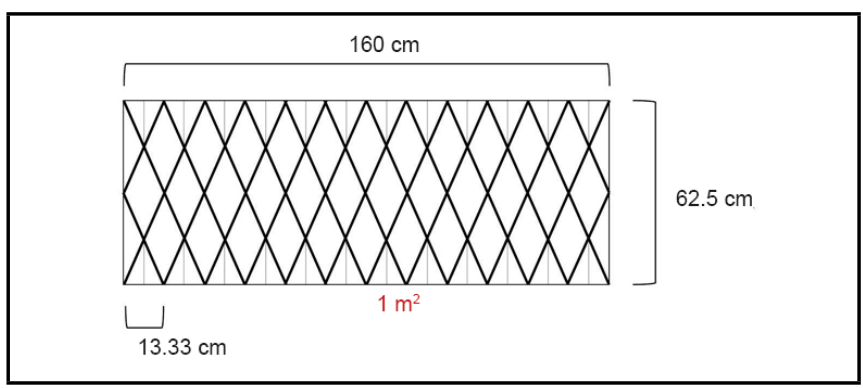

Figure 6. Folding chart of material for producing PSCC shell 3-D PMA.

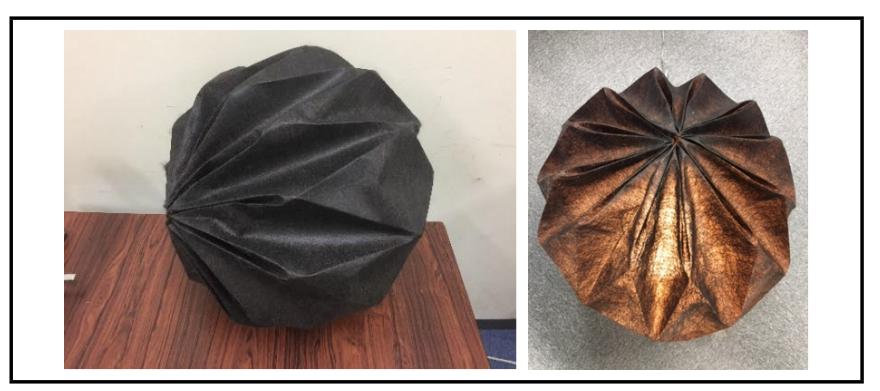

Figure 7. Photographs of specimen PSCC shell 3-D PMA prepared by folding non-woven polypropylene (PP): its appearance (left) and demonstration of light transmissibility when a bulb is installed inside (right).

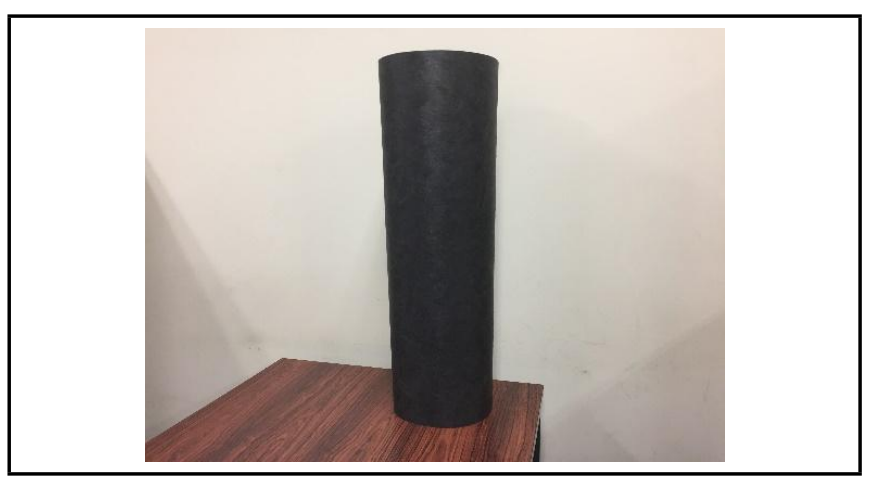

Figure 8. Example of specimen of cylindrical 3-D PMA made with the same material as shown in Fig. 7.

\subsection{Method of Production}

The material was cut to $160 \mathrm{~cm}$ by $62.5 \mathrm{~cm}$ so that the total surface area of the specimen would be $1 \mathrm{~m}^{2}$. Figure 6 shows the folding lines on the material; thick lines indicate mountain folds and thin lines indicate valley folds. After folding according to this figure, top and bottom ends of the material were bound, and left and right ends were bound to each other to make a sphere-like shape. Photographs of the specimen made by the above procedure are presented in Fig. 7.

In order to compare with a cylindrical 3-D PMA (without folding), we also prepared it with the same material and surface area (Fig. 8).

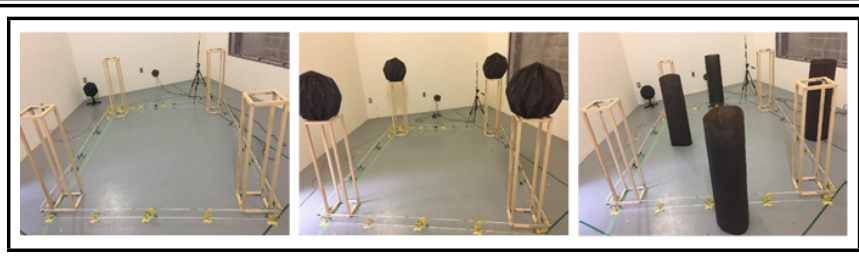

Figure 9. Measurement setup in the reverberation chamber. Empty condition (left), spherical PCCC absorber case (centre), and simple cylindrical absorber case (right).

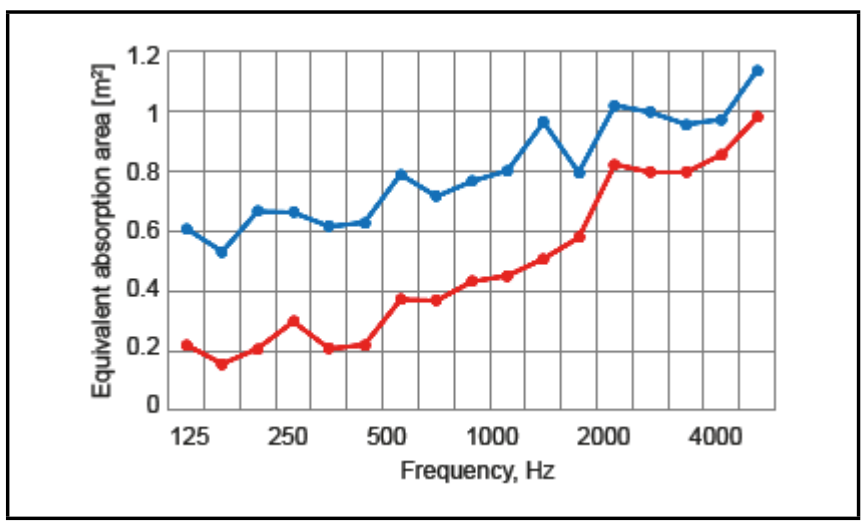

Figure 10. Measured results of total equivalent absorption area for PSCC shell 3-D PMAs (red) compared with cylindrical 3-D PMAs (blue), for four specimens each.

\subsection{Measurement and Measured Results}

The measurement of the reverberation absorption coefficients was performed in a reverberation chamber with $130 \mathrm{~m}^{3}$ volume and $153 \mathrm{~m}^{2}$ surface area. The measurement procedure was principally based on JIS A 1409 (ISO 354 compatible). The measurements were made by a noise interrupting method with one sound source point and three receiving points in the reverberation chamber.

We prepared four specimens for each type of absorber. PSCC shell 3-D PMAs were placed on a wooden stand to be remote from the floor. Cylindrical 3-D PMAs were placed directly on the floor. In order to avoid the effect of sound absorption by the wooden stands, all reverberation time measurements were carried out with the stands staying on the floor (see Fig. 9).

\subsection{Results and Discussion}

The measured results of the equivalent absorption areas for PSCC shell 3-D PMAs are shown in Fig. 10 compared with results of cylindrical 3-D PMAs. Both results are presented in the total equivalent absorption area for four specimens.

The results for both PSCC shell 3-D PMAs and cylindrical 3-D PMAs are not very high. This may be attributed to the low flow resistance of the material used. Therefore, there is a possibility that the absorption performance can be improved if another material of higher flow resistance can be used. Comparing them, the PSCC shell 3-D PMAs showed rather lower absorption performance than the simple cylindrical absorbers, particularly at low frequencies. The PSCC shell 3-D PMA shows quite low absorptivity at low frequencies, but it steeply increases with increasing frequency. This tendency can be related to the surface unevenness due to folding: especially the "valleys" on the PSCC shell surface are quite deep and they have some effect to cause other sound absorbing mechanism than acoustic permeability, e.g., surface viscosity, ${ }^{9}$ etc., which 
were not identified from this experiment.

As theoretical results are not easily obtained for an absorber of complex shape such as a PSCC shell, to make more profound discussion on the absorption mechanism of PSCC shell 3-D PMAs, carrying out experiments that compare PSCC shell 3-D PMAs and spherical absorbers without folding made of the same material will be needed. For the experiment, it is needed to use more flexible and extensible material: the material used in this study was too hard to make a spherical absorber. This is one of the subjects of the future works.

\section{CONCLUDING REMARKS}

In this paper, our pilot studies on the application of a paper folding technique to make uneven surfaces for threedimensional space sound absorbers made of permeable membranes (3-D PMAs) are introduced. First, we conducted a trial to apply the technique to a cylindrical 3-D PMA, which we call PCCC 3-D PMA, as a step toward developing a more designable sound-absorbing lampshade. The measurement results show fairly good performance, almost the same as the simple cylindrical 3-D PMA, provided that the membrane's properties are similar. Next, the trial production of a PSCC 3-D PMA was done. Measurement of its sound absorption performance and a comparison with the cylindrical 3-D PMA was carried out. The results show that the PSCC 3-D PMA proposed in this paper demonstrates comparable sound absorption performance to the simple cylindrical 3-D PMA. However, the absorption performance of the new absorber was not high enough. This is because the flow resistance of the material used is much lower than the optimal value; in this study, for the sake of processability, we used material that is not acoustically optimal. However, the absorptivity can be improved with material of optimal flow resistance. Therefore, both PCCC and PSCC 3-D PMAs can be used not only for sound absorbing lighting equipment but also as a general-purpose sound absorber for better interior design.

Regarding the effect of the shape on the sound absorptivity, i.e., the difference between sphere or cylinder, although the acoustical performance of PSCC 3-D PMAs could be improved by using material with higher flow resistance, the expected advantage of a spherical shape was not confirmed. Also, the effect of the surface unevenness by folding was not identified. These are still open questions and will be the subject of future studies.

\section{ACKNOWLEDGEMENTS}

The authors are indebted to Mr. A. Shimma, and Ms. M. Koeda for their assistance in the experiments.

\section{REFERENCES}

1 Ingard, K. U. Sheet absorbers, in Notes on sound absorption technology, Noise Control Foundation, New York, (1994).

2 Adams, T. Sound Materials: A compendium of sound absorbing materials for architecture and design, Frame Pub., New York, (2017).

3 Takahashi, D., Sakagami, K., and Morimoto, M. Acoustic properties of permeable membranes. J. Acoust. Soc. Am., 99(5), 3003-3009, (1996). https://dx.doi.org/10.1121/1.415213
4 Sakagami, K., Kiyama, M., Morimoto, M., and Takahashi, D. Detailed analysis of the acoustic properties of a permeable membrane, Appl. Acoust., 54(2), 93-111, (1998). https://dx.doi.org/10.1016/S0003-682X(97)00085-6

5 Sakagami, K., Funahashi, K., Somatomo, Y., Okuzono, T., Nishikawa, C., and Toyoda, M. An experimental study on the absorption characteristics a three-dimensional permeable membrane space sound absorber, Noise Control Eng. J., 63(3), 300-307, (2015). https://dx.doi.org/10.3397/1/376327

6 Toyoda, M., Funahashi, K., Okuzono, T., and Sakagami, K. Predicted absorption performance of cylindrical and rectangular permeable membrane space sound absorbers using the three-dimensional boundary element method, Sustainability, 11(9), 2714, (2019). https://dx.doi.org/10.3390/su11092714

7 Sakagami, K., Okuzono, T., Somatomo, Y., Funahashi, K., and Toyoda, M. A basic study on a rectangular plane space sound absorber using permeable membranes, Sustainability, 11(7) 2185, (2019). https://dx.doi.org/10.3390/su11072185

8 Yu, X., Fang, H., Cui, F., Cheng L., and Zhenho, I. Origami-inspired foldable sound barrier designs, J. Sound. Vib., 442, 514-526, (2019). https://dx.doi.org/10.1016/j.jsv.2018.11.025

9 Sakamoto, S., Higuchi, K., and Koseki, S. Study of sound-absorbing materials using layered narrow clearances between two surfaces (Theoretical analysis and experiments of Sensu (folding fan) shaped test samples), Journal of Advanced Mechanical Design Systems and Manufacturing, 9(5), (2015). https://dx.doi.org/10.1299/jamdsm.2015jamdsm0062

10 Suzuki, H. Modified Yoshimura pattern for lighting environment education, J. Geometry and Graphics,20(2), 263271, (2016).

11 Yoshimura, Y. On the mechanism of buckling of a circular cylindrical shell under axial compression, Reports of The Institute of Science and Technology (The University of Tokyo), 5(5), 179-198, (1951).

12 Miura, K. Proposition of pseudo-cylindrical concave polyhedral shells, Report No. 442, Institute of Space and Aeronautical Science, University of Tokyo, 141-163, (1969).

13 Sakagami, K., Oshitani, T., Yairi, M., Toyoda, E., and Morimoto, M. An experimental study on a cylindrical microperforated panel space sound absorber, Noise Control Eng. J., 60(1), 22-28, (2012). https://dx.doi.org/10.3397/1.3670101

14 Sakagami, K., Yairi, M., Toyoda, E., and Toyoda, M. An experimental study on the sound absorption of threedimensional MPP space sound absorbers: rectangular MPP space sound absorber (RMSA), Acoust. Australia, 40(2), 159-162, (2013).

15 LE KLINT web page (online catalogue for LE KLINT $101 \mathrm{XL}$ ), Retrieved from https://www.leklint.com/enGB/Products/Show-Product/LE-KLINT-101XL.aspx, (accessed on 08 August 2019). 